\title{
BLIND CHANNEL IDENTIFICATION FOR MULTIRATE CDMA SYSTEMS
}

\author{
Hongbo Yan and Sumit Roy \\ Dept. of Electrical Engineering \\ University of Washington, Box 352500 \\ Seattle, WA 98195
}

\begin{abstract}
In this paper, we propose a unified approach to blindly identify channels for multirate CDMA systems - both multiple code (MC) and variable processing gain (VPG) systems are considered. A time-domain subspace approach to blind channel estimation introduced in [3] is generalized to the multi-rate case. Performance evaluation is conducted based on the normalized root mean square error (NRMSE) with an appropriate single-rate as a baseline for comparison.
\end{abstract}

\section{INTRODUCTION}

Next generation wireless communication systems will require high-speed access for multimedia services (voice, video and data). Because of the inherent difference in source rates for different services, design of multi-rate communication systems is an imperative. CDMA systems have received much attention for several reasons including the efficient use of radio spectrum. Schemes to extend CDMA to offer multiple data rates are relatively straightforward. Among others, multicode (MC) transmission and variable processing gain (VPG) are the two access methods of particular research interests. Much of the past work has forcused on networking issues [4][5]. Recently, more efforts have been devoted to receiver design[6][7][9]. These techniques are mainly dealing with VPG systems. They often require explicit knowledge of users' signature waveform. This information can be obtained with the aid of training sequences. The price paid is the resultant reduction in channel efficiency. In this paper, we present a blind channel identificaiton algorithm which applies to both VPG or MC multirate systems and single rate systems.

This work was supported in part by in part by AFOSR Grant F49620-1-0472

\section{FORMULATION: VARIABLE PG SYSTEMS}

A baseband VPG multirate CDMA communication system can be modeled as:

$$
x(t)=\sum_{i=1}^{N} \sum_{j=1}^{M_{i}} r_{i, j}(t)+w(t)
$$

where $N$ and $M_{i}$ respectively denote the total number of data rates and the number of users at rate $i, w(t)$ is additive white Gaussian noise. The signal component $r_{i, j}(t)$ due to user $j$ at rate $i$ is given by

$$
r_{i, j}(t)=\sum_{k=-\infty}^{\infty} s_{i, j}(k) g_{i, j}\left(t-k T_{i}\right)
$$

The signature waveform $g_{i, j}(t)$ is defined as the convolution of the unknown channel response $h_{i, j}(t)$ and a known spreading code $\left\{c_{i, j}(l)\right\}$ :

$$
g_{i, j}(t)=\sum_{l=0}^{L_{i}-1} c_{i, j}(l) h_{i, j}\left(t-l T_{c}\right)
$$

where $T_{c}$ is chip duration. Suppose we have

$$
\frac{T_{1}}{p_{1}}=\frac{T_{2}}{p_{2}}=\cdots=\frac{T_{N}}{p_{N}}
$$

where $p_{1}, p_{2}, \cdots, p_{N}$ are co-prime integers, and we assume without loss of generality that $T_{1} \leq \ldots \leq T_{N}$ (resp. $p_{1} \leq \ldots \leq p_{N}$ ). For users that share a common bandwidth, the chip duration must be independent of $i$, and hence we require that

$$
T_{c}=\frac{1}{L} \frac{T_{1}}{p_{1}}=\cdots=\frac{1}{L} \frac{T_{N}}{p_{N}}
$$

where $L$ is some integer greater than 1 . Thus we need an $N$-set of spreading codes, each of length $L_{i}=L p_{i}$ 
for users at different rates. Sampling the received signal at chip rate, we obtain the discrete time model

$$
\begin{gathered}
x(n)=\sum_{i=1}^{N} \sum_{j=1}^{M_{i}} r_{i, j}(n)+w(n) \\
r_{i, j}(n)=\sum_{k=-\infty}^{\infty} s_{i, j}(k) g_{i, j}\left(n-k L_{i}\right)
\end{gathered}
$$

and

$$
g_{i, j}(n)=\sum_{l=0}^{L_{i}-1} c_{i, j}(l) h_{i, j}(n-l)
$$

Generally speaking, the order of channel $h_{i, j}(n)$, $L_{h_{i, j}} \ll L_{i}$, so we reasonably assume that the length of $g_{i, j}(n)$ is $2 L_{i}$ with some zeros at the tail. In matrix form

$$
\begin{gathered}
\mathbf{g}_{i, j}=\left[\begin{array}{c}
g_{i, j}(0) \\
\vdots \\
g_{i, j}\left(2 L_{i}-1\right)
\end{array}\right] \\
=\left[\begin{array}{cccc}
c_{i, j}(0) & 0 & \ldots & 0 \\
\vdots & c_{i, j}(0) & \ddots & \vdots \\
c_{i, j}\left(L_{i}-1\right) & \vdots & \ddots & 0 \\
0 & c_{i, j}\left(L_{i}-1\right) & & c_{i, j}(0) \\
& 0 & \ddots & \vdots \\
\vdots & & \ddots & c_{i, j}\left(L_{i}-1\right) \\
& & & 0 \\
& & & \vdots \\
0 & \ldots & & 0 \\
h_{i, j}(0) \\
\vdots \\
h_{i, j}\left(L_{h_{i, j}}-1\right)
\end{array}\right] \\
\mathbf{C}_{i, j} \mathbf{h}_{i, j}
\end{gathered}
$$

Denote $P=\prod_{i=1}^{N} p_{i}$ and $q_{i}=P / p_{i}$, now we stack the chip-rate samples $r_{i, j}(n)$ collected over an interval of $T=m L_{i} q_{i} T_{\mathrm{c}}=m L P T_{\mathrm{c}}$, where $m$ is defined as smoothing factor. This yields

$$
\begin{aligned}
& \mathbf{r}_{i, j}^{m}(n)=\left[\begin{array}{c}
r_{i, j}\left(n L_{i}\right) \\
\vdots \\
r_{i, j}\left(\left(n+m q_{i}\right) L_{i}-1\right)
\end{array}\right] \\
& =\left[\begin{array}{ccccc}
\mathbf{g}_{i, j}^{2} & \mathbf{g}_{i, j}^{1} & & & \\
& \mathbf{g}_{i, j}^{2} & & \mathbf{0} & \\
& & \ddots & & \\
& \mathbf{0} & \ddots & \mathbf{g}_{i, j}^{1} & \\
& & & \mathbf{g}_{i, j}^{2} & \mathbf{g}_{i, j}^{1}
\end{array}\right] \bullet
\end{aligned}
$$

$$
\begin{aligned}
& {\left[\begin{array}{c}
s_{i, j}(n-1) \\
\vdots \\
s_{i, j}\left(n+m q_{i}-1\right)
\end{array}\right] } \\
=\quad & \mathbf{G}_{i, j} \mathbf{s}_{i, j}(n)
\end{aligned}
$$

where

$$
\mathbf{g}_{i, j}^{1}=\left[\begin{array}{c}
g_{i, j}(0) \\
\vdots \\
g_{i, j}\left(L_{i}-1\right)
\end{array}\right] \quad \mathbf{g}_{i, j}^{2}=\left[\begin{array}{c}
g_{i, j}\left(L_{i}\right) \\
\vdots \\
g_{i, j}\left(2 L_{i}-1\right)
\end{array}\right]
$$

Therefore, the observation during the interval can be expressed as

$$
\begin{aligned}
& \mathbf{x}_{K}=\left[\begin{array}{c}
x(K L P) \\
\vdots \\
x(K L P+L P-1)
\end{array}\right] \\
& =\sum_{i=1}^{N} \sum_{j=1}^{M_{i}} \mathbf{r}_{i, j}\left(K q_{i}\right)+\mathbf{w}_{K} \\
& \left.=\quad \mathbf{G}_{1,1} \cdots \mathbf{G}_{N, M_{N}}\right]\left[\begin{array}{c}
\mathbf{s}_{1,1}\left(K q_{1}\right) \\
\vdots \\
\mathbf{s}_{N, M_{N}}\left(K q_{N}\right)
\end{array}\right] \\
& =\mathbf{G s}_{K}+\mathbf{w}_{K}
\end{aligned}
$$

Now we can construct a data matrix $\mathbf{X}$ by placing $J$ successive $\mathbf{x}_{K}$ 's side by side

$$
\begin{aligned}
& \mathbf{X}=\left[\begin{array}{lll}
\mathbf{x}_{K} & \cdots & \mathbf{x}_{K+J-1}
\end{array}\right] \\
& =\mathbf{G}\left[\mathbf{s}_{K} \cdots \mathbf{s}_{K+J-1}\right]+\left[\begin{array}{lll}
\mathbf{w}_{K} & \cdots & \mathbf{w}_{K+J-1}
\end{array}\right] \\
& =\mathbf{G S}+\mathbf{W}
\end{aligned}
$$

The problem addressed in this paper is the estimation of $h_{i, j}(n)$ 's from $\mathbf{X}$ without the knowledge of $\mathbf{S}$.

\subsection{Single Rate and Multi-Rate MC systems}

It is easy to see that single rate is a special case of the VPG multirate systems with $p_{1}=p_{2}=\cdots=p_{N}=$ 1 , and $M_{1}=M_{2}=\cdots=M_{N}=1$, for which the snapshot interval $T=m L T_{c}$. The matrix $\mathbf{G}$ therefore has dimension $m L \times(m+1) N$.

In MC systems, multirate is implemented as an aggregation or multiple of several data streams at some basic rate [5]. Generally, the basic symbol duration $T_{b r}$ is define from (4) as

$$
\frac{T_{b r}}{P}=\frac{T_{1}}{p_{1}}=\frac{T_{2}}{p_{2}}=\cdots=\frac{T_{N}}{p_{N}}
$$

A high-rate user at $p$ times the basic rate, is converted into $p$ basic rate streams via subsampling. Each 
low-rate stream is time-spread with mutually orthognal codes which are then superimposed before transmission. Obviously in MC systems, high-rate users are decomposed into several virtual users all at the same basic rate. In this manner, MC systems are converted into an equivalent single rate system and hence our formulation still applies.

\subsection{Extracting infomation about $G$ from $X$}

From the knowledge of linear algebra, we know that if $\mathbf{S}$ has full row rank and $\mathbf{G}$ has full column rank, then $\mathbf{X}$ has the same column space as $\mathbf{G}$ [1]. The necessary condition for the full row rank property of $\mathbf{S}$ is the number of columns being greater than the number of rows, i.e. $J>\sum_{i=1}^{N} M_{i}\left(m q_{i}+1\right)$. This can be easily satisfied by accumulating data vectors for a long enough interval. We notice that once this condition is satisfied, the randomness of $s_{i, j}(k)$ 's will generally guarantee the desired property of $\mathbf{S}$. Similarly, the necessary condition for the full column rank property of $\mathbf{G}$ is the number of rows being greater than the number of columns, indicating

$$
m L P>\sum_{i=1}^{N} M_{i}\left(m q_{i}+1\right)
$$

An interesting observation is that for single rate systems, (14) reduces to $m L>N(m+1)$, which means at most $N=m L /(m+1)$ users can be accommodated in the system. However for MC systems (14) yields $m L>\sum_{i=1}^{N} M_{i} q_{i}(m+1)$. Obviously, by choosing appropriate $M_{i}$ 's, more users may be accommodated in either MC or VPG systems for given $m L P$.

\section{ALGORITHM DEVELOPMENT}

Suppose the necessary condition elaborated above is satisfied. Applying the SVD to data matrix $\mathbf{X}$, we have

$$
\mathbf{X}=\left[\begin{array}{ll}
\mathbf{U}_{s} & \mathbf{U}_{w}
\end{array}\right]\left[\begin{array}{cc}
\boldsymbol{\Lambda}_{s} & \mathbf{0} \\
\mathbf{0} & \boldsymbol{\Lambda}_{w}
\end{array}\right]\left[\begin{array}{l}
\mathbf{V}_{s}^{H} \\
\mathbf{V}_{w}^{H}
\end{array}\right]
$$

The vectors in $\mathbf{U}_{s}$, associated with the singular values in diagonal matrix $\boldsymbol{\Lambda}_{s}$, span the signal space defined by the columns of $\mathbf{G}$, and its complement $\mathbf{U}_{w}$, associated with the 'noise' singular values in diagonal matrix $\boldsymbol{\Lambda}_{s}$ span the noise space. Thus we have

$$
\mathbf{U}_{w}^{H} \mathbf{G}=\mathbf{0}
$$

Since $\mathbf{G}=\left[\ldots \mathbf{G}_{i, j} \ldots\right]$, this yields

$$
\mathbf{U}_{w}^{H} \mathbf{G}_{i, j}=\mathbf{0}
$$

(17) is equivalent to

$$
\tilde{\mathbf{U}}_{w}^{H} \mathbf{g}_{i, j}=\tilde{\mathbf{U}}_{w}^{H} \mathbf{c}_{i, j} \mathbf{h}_{i, j}=0
$$

where

$$
\begin{gathered}
\tilde{\mathbf{U}}_{w}=\left[\begin{array}{ccccc}
\mathbf{0} & \mathbf{U}_{w, 1} & \mathbf{U}_{w, 2} & \ldots & \mathbf{U}_{w, m q_{i}} \\
\mathbf{U}_{w, 1} & \mathbf{U}_{w, 2} & \cdots & \mathbf{U}_{w, m q_{i}} & \mathbf{0}
\end{array}\right] \\
\mathbf{U}_{w}=\left[\begin{array}{c}
\mathbf{U}_{w, 1} \\
\vdots \\
\mathbf{U}_{w, m q_{i}}
\end{array}\right] \quad 1 \leq l \leq m q_{i}
\end{gathered}
$$

since

$$
\mathbf{U}_{w}^{H} \mathbf{G}_{i, j}=\left[\begin{array}{c}
\mathbf{U}_{w, 1}^{H} \mathbf{g}_{i, j}^{2} \\
\mathbf{U}_{w, 1}^{H} \mathbf{g}_{i, j}^{1}+\mathbf{U}_{w, 2}^{H} \mathbf{g}_{i, j}^{2} \\
\vdots \\
\mathbf{U}_{w, m q_{i}-1}^{H} \mathbf{g}_{i, j}^{1}+\mathbf{U}_{w, m q_{i}}^{H} \mathbf{g}_{i, j}^{2} \\
\mathbf{U}_{w, m q_{i}}^{H} \mathbf{g}_{i, j}^{1}
\end{array}\right]=\tilde{\mathbf{U}}_{w}^{H} \mathbf{g}_{i, j}
$$

To avoid the trivial solution $\mathbf{h}=\mathbf{0}$, we determine $\mathbf{h}_{i, j}$ by

$$
\hat{\mathbf{h}}_{i, j}=\arg \min _{\left\|\mathbf{h}_{i, j}\right\|=1}\left\|\tilde{\mathbf{U}}_{w}^{H} \mathbf{c}_{i, j} \mathbf{h}_{i, j}\right\|^{2}
$$

\section{SIMULATION RESULTS}

Simulations were conducted to compare the performance of the algorithm for the single-rate system vis-a-vis multi-rate MC and VPG systems. For a fair comparison, we established the follow baseline:

1. Same system bandwidth, i.e., chip duration is identical for all the three systems;

2. Same rate budget - net data rate is conserved, i.e.

$$
\frac{M_{1}}{T_{1}}+\cdots+\frac{M_{N}}{T_{N}}=\frac{M_{s r}}{T_{s r}}
$$

where $T_{s r}$ and $M_{s r}$ are the symbol duration and number of users for single rate systems, the left side of the equation applies to both VPG and MC multirate systems;

3. Same total number of symbols used in the simulation;

4. Same snapshot duration, i.e. number of rows in the matrix $\mathbf{X}$ is identical in all cases.

The performance is evaluated in terms of normalized root mean-square error (NRMSE), defined as

$$
N R M S E=\frac{1}{\|\mathbf{h}\|} \sqrt{\frac{1}{D} \sum_{d=1}^{D}\left\|\hat{\mathbf{h}}_{d}-\mathbf{h}\right\|^{2}}
$$

where $\hat{\mathbf{h}}_{d}$ is the channel estimation at the $d$ th run, $\mathbf{h}$ is real channel and $D$ is total number of Monte-Carlo runs. The two-ray mutipath channel

$$
h(t)=\left[c(t)-0.7 c\left(t-T_{c} / 3\right)\right] W_{6 T_{c}}
$$




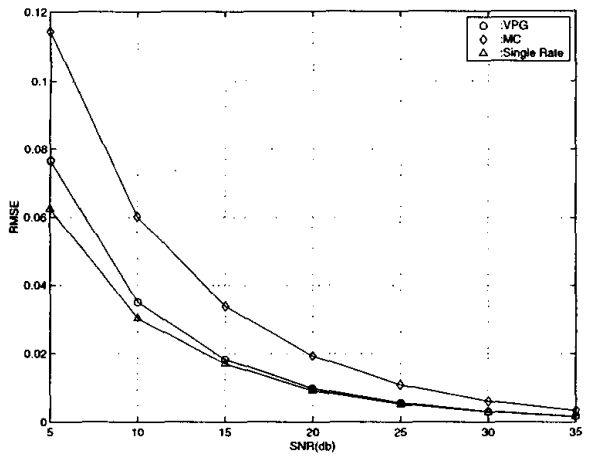

Figure 1: NRMSE versus SNR

is used where $c(t)$ is raised-cosine pulse function limited to $6 T_{c}$ with roll-off factor 0.10 . To eliminate the effect of the possible spreading code dependency [8], we randomly generated the codes in the simulations.

Example 1: We consider the dual rate case where the ratio of symbol rate is $2 / 3$, i.e. $p_{1}=2, p_{2}=3$. The number of users at each rate is 1 . In VPG systems, choose $L=15$, thus the processing gains for rate 1 and rate 2 are 30 and 45 respectively. Smoothing factor $m$ is set to be 2. Data obtained within the duration $50 p_{1} T_{2}=50 p_{2} T_{1}$ (therefore 150 symbols transmitted at rate 1,100 symbols sent at rate 2) were used in each independent trial. Under the baseline described above, we obtained the corresponding settings for MC systems as: processing gain 90 , smoothing factor 2, 50 symbols transmitted by each of the 5 virtual users; and for single rate systems as: processing gain 36 ; smoothing factor 5,125 symbols transmitted by each of the two users. 50 independent runs were conducted to compute the average NRMSE over users for each system. The result is shown in figure 1.

Example 2: Under the same settings described above, we performed simulations with different total number of symbols at $S N R=5 \mathrm{db}$. The result is shown in figure 2.

From Figs. 1, 2 we can see that single rate systems have the best performance among the three, with signficant differences at low SNR or small number of symbols. Among the two multi-rate systems, the VPG is clearly superior to $\mathrm{MC}$ and performs close to the single-rate case.

\section{REFERENCES}

[1] I. Satake, Linear Algrbra, New York: Marcel Dekker Inc. 1975.

[2] H. Liu and G. Xu, "A subspace method for signa-

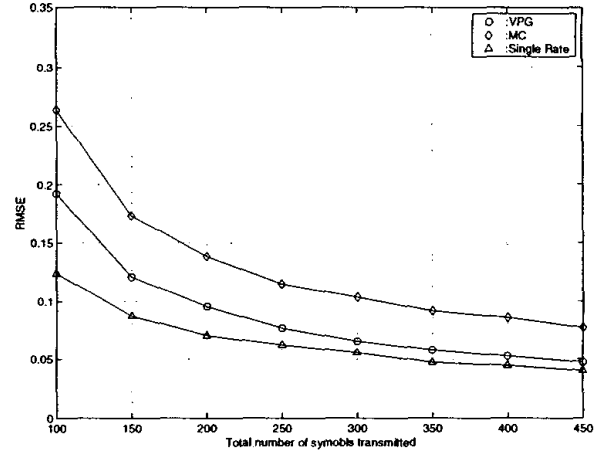

Figure 2: NRMSE versus total number of symbols transmitted, $\mathrm{SNR}=5 \mathrm{db}$

ture waveform estimation in synchronous CDMA systems", IEEE Trans. Commun., vol.44, no.10, pp. 1346-1354, Oct. 1996.

[3] M.Torlak and G.Xu, "Blind multiuser channel estimation in asynchronous CDMA systems", IEEE Trans. Signal Processing, Vol.45, no.1, pp.137-147, Jan. 1997.

[4] C-L. I and K.K. Sabnani, "Variable spreading gain CDMA with adaptive control for true packet switching wireless network," in Proc. ICC, vol.2, pp.725-730, June 1995.

[5] C-L. I and R.D. Gitlin, "Multi-Code CDMA wireless personal communications networks", in Proc. ICC, vol.2, pp.1060-1064, June 1995.

[6] A.-L. Johansson and A. Svensson, "Successive interference cancellation in multiple data rate DS/CDMA systems," in Proc. VTC, vol.2, pp.704708, July 1995.

[7] J. Chen and U. Mitra, "Further results for multirate decorrelators for synchronous DS/CDMA systems," in Proc. 34th Annual Allerton Conf. Commun., Contr., and Comput., pp.170-179, Oct.1996.

[8] U.Mitra, "Comparison of maximum likelihoodbased detection for two multi-rate access schemes for CDMA signals," IEEE Trans. Commun. vol.47, no.1, pp64-77, Jan. 1999.

[9] M. Saquib, R. Yates and N. Mandayam, "Decorrelating detectors for a dual rate synchronous DS/CDMA system", Wireless Personal Commun., vol.9, no.3, pp.197-216, May 1999. 